\title{
Target preparation for neutron-induced reaction measurements
}

\author{
Goedele Sibbens*, Alf Göök, David Lewis, André Moens, Stephan Oberstedt, David Vanleeuw, Ruud \\ Wynants, and Mariavittoria Zampella
}

European Commission, Joint Research Centre, Directorate G, Retieseweg 111, B-2440 Geel, Belgium

\begin{abstract}
Neutron-induced reaction measurements, e.g. cross-sections and particle emission yields, require samples, called "targets", with specific properties depending on the reaction being studied and the quantities being measured. The target characteristics influence the results of these measurements and can have a strong impact on the total uncertainty of neutron-induced reaction data, which are important for industry and in research. This paper gives an overview of the main techniques applied in the target preparation laboratory at JRC-Geel for production and characterization of targets for neutron-induced reaction data measurements. The use of different targets is demonstrated with a few examples of total and reaction cross-section as well as nuclear fission experiments. In addition, on-going investigations and technical developments are presented.
\end{abstract}

\section{Introduction}

High-quality and well-characterized samples, called "targets", are one of the pillars of nuclear data experiments together with the nuclear facilities, the data acquisition systems, and the theoretical and numerical data processing. Nuclear targets consist of a freestanding or a supported material on a substrate and range in thickness from millimetres to nanometres. They have to be physically and chemically stable. The target substrate is as thin as reasonably possible, mechanically stable and should not undergo any nuclear reaction leading to products that interfere with the material under investigation.

The data is generated by measurements at the two large-scale neutron facilities at the JRC-Geel. Both facilities rely on accelerators which provide charged particle beams that in turn produce neutrons through nuclear reactions. At GELINA a $150 \mathrm{MeV}$ linear accelerator produces an electron beam which hits a rotating uranium disc. When the beam hits the uranium disc, Bremsstrahlung is produced, leading to neutrons via $(\gamma, \mathrm{n})$ and to a lesser extent $(\gamma, \mathrm{f})$ reactions. At MONNET, a 3.5 MV light-ion Tandem accelerator which replaced recently the $7 \mathrm{MV}$ light-ion Van de Graaff accelerator, provides charged particle beams, which allow producing quasi mono-energetic neutrons up to $20 \mathrm{MeV}$ through reactions between a suitable particle beam and a neutron-producing target [1, 2]. Already from the 60's the CBNM (Central Bureau for Nuclear Measurements), renamed IRMM and later more recently JRC-Geel, was preparing targets for nuclear physicists who were measuring neutron-induced reaction cross-sections as recommended on the priority list of the EANDC (European-American Nuclear Data Committee) which was later reorganised to form the NEA (Nuclear Energy Agency) of the OECD (Organisation for Economic Co-operation and Development) [3, 4]. The main users of these nuclear data are European Union member state organisations and international partners of the EURATOM program, in particular European safety authorities, the nuclear industry, the nuclear research community, the OECD NEA and the IAEA (International Atomic Energy Agency) [1]. A history of the techniques applied at JRC-Geel to prepare and characterize targets is described in $[5,6]$.

This paper describes the main techniques applied nowadays in the target preparation laboratory at JRCGeel for the production and characterization of nuclear targets. The use of these targets is demonstrated with a few examples of neutron cross-section measurements. In addition, ongoing investigations related to molecular plating, pellet compacting, e-beam evaporation, cleaning of glass plates for the production of polyimide foils and vacuum deposition parameters are presented.

\section{Neutron-induced reactions}

Neutron-induced reactions are of importance for various applications, e.g. energy production and medical treatment. For the quantification of the energy production in a nuclear reactor or the applied dose to the tissue in the human body we need to know the crosssections for the various reactions a neutron may undergo with different elements (isotopes) present in a nuclear reactor or a human body, respectively. Cross-sections and secondary particle emission production yields, e.g. alpha particles, gamma-rays, secondary neutrons and fission fragments, need to be known as a function of the incident neutron energy. Dedicated measurement

* Corresponding author: goedele.sibbens@ec.europa.eu 
programmes are being executed at the two JRC neutron sources (GELINA, MONNET) since more than 50 years.

Different types of neutron-induced reactions will lead to different reaction products, e.g. neutrons from elastic and inelastic scattering reactions ${ }^{\mathrm{A}} \mathrm{X}(\mathrm{n}, \mathrm{n}){ }^{\mathrm{A}} \mathrm{X}$ and ${ }^{\mathrm{A}} \mathrm{X}\left(\mathrm{n}, \mathrm{n}^{\prime} \gamma\right)^{\mathrm{A}} \mathrm{X}$ respectively, photons from radiative capture reactions ${ }^{\mathrm{A}} \mathrm{X}(\mathrm{n}, \gamma){ }^{\mathrm{A}+1} \mathrm{X}$ or fission fragments from neutroninduced fission reactions [7]. Other neutron absorption processes can lead to proton or alpha particle emission. Fig. 1 shows a schematic overview of a number of possible neutron-induced reactions.

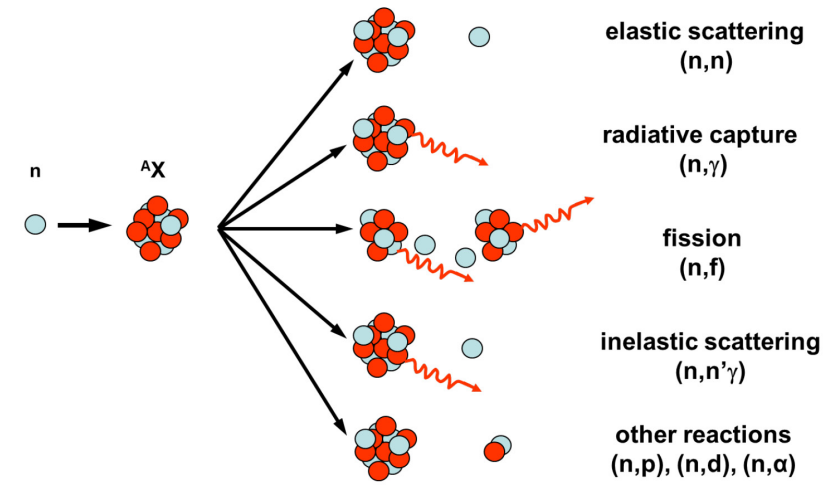

Fig. 1. Neutron-induced reactions.

The neutron cross-section is a measure of the probability for an interaction between an incident neutron and a target nucleus. Neutron cross-section measurements can be divided into transmission measurements and reaction cross-section measurements. In transmission measurements the observed quantity is the fraction of the neutron beam that travels through the target without any interaction. Targets for transmission measurements have to be homogeneous without holes and covering a range of thicknesses. As a general rule, a transmission between 0.2 and 0.7 , depending on the background conditions, can be recommended. To ensure the homogeneity of the target, for some elements, measurements are performed on powder mixtures (with sulphur or carbon) or solutions. In reaction cross-section measurements the observed quantity is the yield which is the fraction of the neutron beam that undergoes a reaction in the target. For reaction cross-section measurements in which the escape probability of the reaction product is strongly influenced by the target and/or the substrate thickness, targets are preferably thin homogeneous layers on thin backings [8].

For the understanding of the nuclear fission process the fission fragment production yields, the mass-resolved kinetic energy distribution and the characteristics of the particles, neutrons and gamma-rays, emitted from the highly excited fragments shortly after scission of the fissioning nucleus needs to be known. Usually two different techniques are applied to measure fission fragment emission yields, either recoil massspectrometry [9] or the double-energy/double-velocity technique [10-12]. Pre-requisite for precise measurements performed with either of the two techniques is to use very thin and homogenous actinide deposits to minimize the energy loss of the fission fragments when passing that layer. For the doubleenergy/double-velocity technique it is important that the substrate, onto which the very thin actinide layer is deposited, is thin and homogenous, too. In the ideal case the energy loss should be so small that light and heavy fragments are separated, independent of the path length which the ion has to pass through the target or the target and the substrate layer.

In general for neutron-induced reactions the areal density of the nuclide under investigation needs to be known. In addition, the homogeneity of the target and the presence of contaminants and impurities, which can influence the measurement, need to be checked.

\section{Target preparation characterization of thick targets}

and

\subsection{Mechanical transformation}

Several mechanical transformation techniques are applied to re-shape material. In the case of radioactive material, the re-shaping is done in a glove box in the nuclear controlled area. For oxidising material an argon glove box is used as far as possible, otherwise the material is protected with an oil or petrol layer from reaction with the environment. A piece of metallic material can be transformed into a thin foil with a thickness between $1.0 \mathrm{~mm}$ and $0.05 \mathrm{~mm}$ by rolling and, afterwards, into a disc with a diameter ranging from $1 \mathrm{~mm}$ to $100 \mathrm{~mm}$ and a thickness up to $0.5 \mathrm{~mm}$ by punching. Metallic wires can be produced with a diameter down to $0.5 \mathrm{~mm}$ by wire drawing.

\subsection{Powder compacting}

Powder targets are prepared by canning the powder in an Al container in which the powder is slightly manually pressed and the cover of the Al can is glued. In case of pellets the powder is mixed with sulphur powder and cold compacted with a hydraulic press. Sulphur has been chosen as binder for form stability. The required pressure to prepare pellets depends on the diameter of the matrix and the die. In case of radioactive material, the preparation of powder targets is performed in a glove box in the nuclear controlled area.

\subsection{Characterization}

Metal targets like discs and foils are characterised for their mass and dimensions. The mass is determined by accurate weighing with a microbalance applying the substitution method. In substitution weighing, the mass of the target is determined via the so called "SUUS" method in which a series of mass determinations of an unknown (U) and a reference weight (S) is performed. The uncertainty contributions are the uncertainties associated with the calibrated reference weights, the air buoyancy correction and the repeatability of the balance. The diameter of the targets is measured accurately using a non-contact vision measuring microscope based on 
leading-edge optical technologies with a readability of $0.5 \mu \mathrm{m}$. The contour of the target is scanned several times at both sides. In case of a powder pellet the diameter is not measured directly, instead the inside diameter of the press tool is taken. The thickness measurements are done with a calliper, in case of powder pellets, or a thickness gauge for the other targets. Before each set of measurements the measurement instrument is calibrated with reference gauge blocks. The uncertainty contributions are the uncertainties coming from the reference gauge blocks and the repeatability of the measurements.

Table 1 gives an overview of present preparation and characterization techniques for thick targets.

Table 1. Overview of the present preparation and characterization techniques for thick targets.

\begin{tabular}{|c|c|c|}
\hline Method $\backslash$ Material & Metal & Powder \\
\hline $\begin{array}{c}\text { Mechanical } \\
\text { transformation } \\
\text { Powder compacting }\end{array}$ & $\begin{array}{c}\text { Cutting, rolling, } \\
\text { punching and } \\
\text { drawing equipment }\end{array}$ & Hydraulic press \\
\hline $\begin{array}{c}\text { Characterization } \\
\text { Weighing } \\
\begin{array}{c}\text { Area's and } \\
\text { thickness } \\
\text { measurement }\end{array}\end{array}$ & $\begin{array}{c}\text { Vision measuring } \\
\text { microscope and } \\
\text { thickness gauge }\end{array}$ & Balance \\
\hline
\end{tabular}

\section{Target preparation and characterization of thin targets}

\subsection{Thin foil preparation}

For neutron-induced reaction measurements where the energy loss of the reaction product in the substrate needs to be minimized, thin $\mathrm{Al}$ foils with a thickness of 25 to $30 \mu \mathrm{m}$ on an Al ring can be used. Fig. 2 shows the stretching process of the $\mathrm{Al}$ foil. The foil is first locked between two rings and then placed on a support for stretching. The Al rings are glued on the stretched Al foil and later cut out.
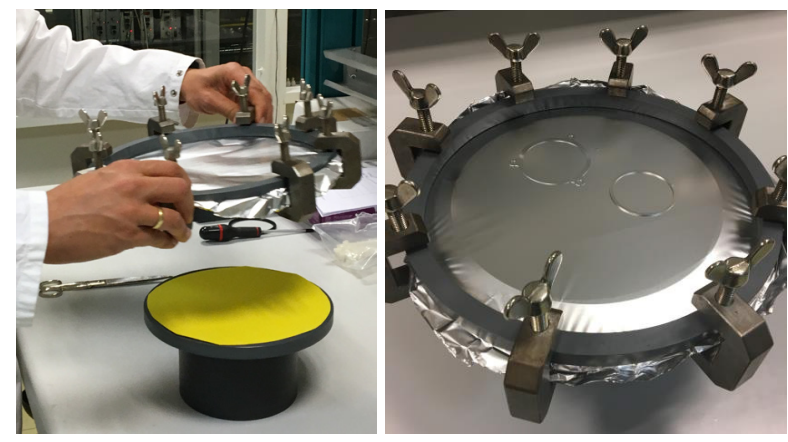

Fig. 2. Stretching process of an $\mathrm{Al}$ foil.

Another type of thin substrate is a polyimide foil with a typical areal density of $35 \mu \mathrm{g} \cdot \mathrm{cm}^{-2}$ produced by in-situ polymerization [13]. The first two steps of this method, which are the preparation of the polycondensate solution and the glass coating, are done in an argon glove box to keep the relative humidity lower than $15 \%$. The third step which is the polymerization is performed at $350^{\circ} \mathrm{C}$ in a dust-free oven. Finally the foils are cut on the glass plate and transferred on a frame via a water surface in a laminar flow cabinet. The polyimide foil can be coated afterwards with a $35 \mu \mathrm{g} \cdot \mathrm{cm}^{-2}$ Au layer by physical vapour deposition to become electrically conductive.

\subsection{Thin foil characterization}

The areal density of the polyimide foils is measured with a visible light spectrophotometer. With this instrument the amount of light is measured that is refracted and/or reflected from the top and the bottom of the polyimide foil as function of the wavelength. The interference pattern is used to calculate the foil thickness. When the foil is still on the glass plate, the light reflection mode is applied. After transferring the foil on a frame, the areal density is re-measured via the light transmission mode [13]. The relative uncertainty on the areal density is in the order of $10 \%$.

\subsection{Thin film deposition}

Thin film deposits with an areal density ranging from 10 to $300 \mu \mathrm{g} \cdot \mathrm{cm}^{-2}$ are prepared by two kinds of techniques: physical vapour deposition and the so-called "molecular plating". In case the ingrowth of a daughter nuclide would interfere with the characterization and neutroninduced reaction measurements, the actinide material is purified prior to the deposition on a substrate. The radiochemical separation is performed by ion exchange [14].

\subsubsection{Physical vapour deposition}

${ }^{235,238} \mathrm{U}, \mathrm{Li}, \mathrm{B}$, tristearin and $\mathrm{Au}$ layers are prepared by physical vapour deposition in different set-ups, dedicated to each material to avoid cross-contamination. The principle of this system is to evaporate or sublimate the starting material in a vacuum and condense the vapour on a substrate.

The evaporators, except for $\mathrm{B}$, consist of a Ta crucible connected to a power supply in a closed chamber, connected to a pump system in order to work under vacuum conditions as described in [16]. The distance between the material to be evaporated and the substrates is determined according to the traveling distance of the evaporated atom or molecule, the required homogeneity and yield and is set at $195 \mathrm{~mm}$ in the evaporators for ${ }^{235,238} \mathrm{U}$, at $400 \mathrm{~mm}$ for $\mathrm{Li}$, at $200 \mathrm{~mm}$ for tristearin and at $250 \mathrm{~mm}$ for $\mathrm{Au}$. $\mathrm{Al}$ rings are mounted as masks in front of the substrates and determine the requested area of the deposited material. The deposit thickness is monitored with a quartz crystal microbalance during the deposition process.

For the evaporation of ${ }^{10} \mathrm{~B}$, which has a melting point of $2349 \mathrm{~K}\left(2076^{\circ} \mathrm{C}\right)$, a bent electron beam (e-beam) bombardment source is used for the heating of the $\mathrm{B}$ material. In this case the evaporation rate is controlled 
by the e-beam current. The old e-beam evaporator for B has been replaced by a new one which has multiple water cooled cupper pockets with a tungsten liner for the source material. The deposition rate is monitored with a quartz crystal microbalance. The distance between the evaporation source and the substrate can be changed from 300 to $400 \mathrm{~mm}$. Prior to a deposition the substrate can be plasma cleaned with a glow discharge module. The equipment has the possibility to heat the substrate during and after deposition. More information of the new e-beam evaporator including $\mathrm{B}$ evaporation tests and calibration of the thickness monitor by weighing and neutron time of flight measurements has been described by Vanleeuw et al. in [15].

To prepare deposits of ${ }^{235} \mathrm{U}$ [16] and ${ }^{238} \mathrm{U}$ [17] by resistance heating in vacuum, the $\mathrm{U}_{3} \mathrm{O}_{8}$ starting material needs first to be converted into $\mathrm{UF}_{4}$ by a wet chemical precipitation method because $\mathrm{U}_{3} \mathrm{O}_{8}$ will be reduced into more stable $\mathrm{UO}_{2 \pm \mathrm{x}}$ when heated in vacuum and so temperatures much higher than the $\mathrm{U}_{3} \mathrm{O}_{8}$ melting point of $1423 \mathrm{~K}$ are needed for the deposition process which is not the case for $\mathrm{UF}_{4}$. Each uranium evaporator is integrated in a glove box in the nuclear controlled area.

The vacuum deposition of metallic $\mathrm{Li}$ is performed in a multi-crucible evaporation set-up that consists of a tantalum crucible heater surrounding an alumina crucible for the $\mathrm{Li}$ material. A second crucible of tantalum is present to be able to evaporate a $50 \mu \mathrm{g} \cdot \mathrm{cm}^{-2} \mathrm{Au}$ passivation layer on the substrate prior to the $\mathrm{Li}$ deposition and a $50 \mu \mathrm{g} \cdot \mathrm{cm}^{-2}$ Au protective layer coating the lithium deposit directly after evaporation to avoid that $\mathrm{Li}$ reacts with the substrate and the environmental elements, respectively [18].

The above mentioned techniques are applied one after another in case of a multi-layer target [19].

\subsubsection{Molecular plating}

The molecular plating technique is based on the cathodic deposition of the actinide material onto an Al substrate in isopropanol at a constant voltage of $360 \mathrm{~V}$. For each isotope of $\mathrm{U}, \mathrm{Pu}, \mathrm{Am}$ and $\mathrm{Np}$ a molecular plating cell is designed and produced in the JRC-Geel workshop according to the required dimensions of the substrate and the deposit. The anode is a platinum grid rotating at a speed of 5 to 10 rounds per minute. The cathode is connected to a stainless steel holder which contains the $\mathrm{Al}$ substrate with an $\mathrm{Al}$ ring as mask that determines the diameter of the actinide deposit. Prior to the deposition the $\mathrm{Al}$ substrate is chemically etched in a mixture of $80 \% \mathrm{H}_{3} \mathrm{PO}_{4}+4 \% \mathrm{HNO}_{3}+16 \% \mathrm{H}_{2} \mathrm{O}$. The original oxide material is first dissolved in $14 \mathrm{M} \mathrm{HNO}_{3}$, evaporated to near dryness and subsequently re-dissolved in $0.75 \mathrm{M}$ $\mathrm{HNO}_{3}$ at a concentration linked to the required plated amount on the substrate. Volumes of 20 up to $100 \mu \mathrm{L}$ of the actinide nitrate solution are added to $20 \mathrm{~mL}$ up to $200 \mathrm{~mL}$ of isopropanol depending on the volume of the cell. After two hours of molecular plating the $\mathrm{Al}$ substrate with deposit is removed from the cell and heated up on a hotplate for 5 minutes at $120^{\circ} \mathrm{C}$ to evaporate the remaining isopropanol [20, 21]. To avoid that dust can enter via the top part and to re-load and empty the cell in a faster and safer way, the upper part has recently been redesigned. Fig. 3 shows pictures of the new molecular plating cell.

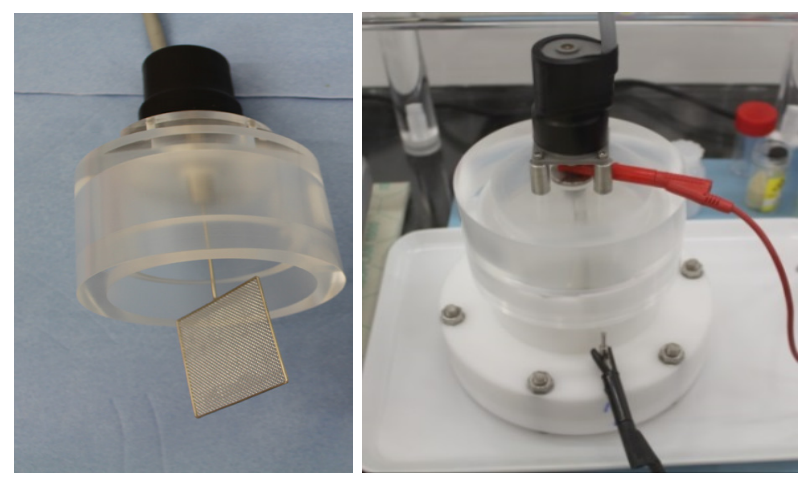

Fig. 3. Pictures of the molecular plating cell; left part: new upper part with Pt anode, right part: cell connected to power supply.

\subsection{Characterization}

The total activity of the actinide layers is determined by low-geometry alpha-particle counting [22]. The measurements are carried out using two different setups: an alpha-particle counter with variable $(50-200 \mathrm{~mm})$ and with fixed $(1637 \mathrm{~mm})$ target-to-detector distance as described in [14]. The solid angle is determined by the distance of the target to the diaphragm in front of the detector, the diameter of the diaphragm and of the deposited layer. The latter is not measured directly; instead the inner diameter of the mask, used during the physical vapour deposition or molecular plating, is measured. For the alpha counter with variable length, the distance from the target to the diaphragm in front of the detector is measured with calibrated gauge blocks. From the measured activity, the isotopic composition and deposited area, the mass and areal density of the isotope of interest is calculated. The uncertainty contributions are the uncertainties associated with the counting statistics, the corrections for background, for decay, for ingrowth of daughter nuclide, the measurement time, the isotopic composition and the solid angle.

A morphological and compositional study of ${ }^{238} \mathrm{U}$ thin film targets showed that layers prepared by vacuum deposition are smooth $\mathrm{UF}_{4}$ deposits [21]. Results of homogeneity measurements showed a variation of the areal density of the order of $5 \%$ between the centre and the edge of deposits with a diameter of $70 \mathrm{~mm}$ [22]. The targets prepared by molecular plating are maze-like layers of U(VI) co-deposited with isopropanol [21].

The mass of stable deposits is determined by difference weighing. Therefore, the mass of the substrate is measured on a microbalance before and after deposition by means of the substitution method. The areal density is calculated from the mass, corrected for isotopic abundance and impurities, and the diameter of the deposit that is determined by measurement of the inner diameter of the mask used during the physical vapour deposition. The uncertainty contributions are the uncertainties associated with the calibrated reference 
Table 2. Overview of the present preparation and characterization techniques for thin targets.

\begin{tabular}{|c|c|c|c|c|c|c|}
\hline Method $\backslash$ Material & ${ }^{235} \mathbf{U},{ }^{238} \mathbf{U}$ & $\begin{array}{c}\text { U, Pu, } \\
\text { Am, Np }\end{array}$ & $\begin{array}{c}\text { LiF } \\
\text { Metallic } \\
\text { Li }\end{array}$ & $\mathrm{C}_{57} \mathrm{H}_{110} \mathrm{O}_{6}$ & Au & ${ }^{10} B$ \\
\hline $\begin{array}{c}\text { Preparation } \\
\text { Vacuum deposition } \\
\text { Molecular plating } \\
\end{array}$ & $\begin{array}{l}\text { Resistance } \\
\text { Isopropanol }\end{array}$ & Isopropanol & Resistance & Resistance & Resistance & e-beam \\
\hline $\begin{array}{c}\text { Characterization } \\
\text { Weighing } \\
\text { Area's measurement } \\
\text { a-particle counting } \\
\text { Combustion analysis }\end{array}$ & $\begin{array}{c}\text { Calliper } \\
\text { Low geometry }\end{array}$ & $\begin{array}{c}\text { Calliper } \\
\text { Low geometry }\end{array}$ & $\begin{array}{l}\text { Balance } \\
\text { Calliper }\end{array}$ & $\begin{array}{c}\text { Balance } \\
\text { Calliper } \\
\text { in } \mathrm{O}_{2}\end{array}$ & $\begin{array}{l}\text { Balance } \\
\text { Calliper }\end{array}$ & $\begin{array}{l}\text { Balance } \\
\text { Calliper }\end{array}$ \\
\hline
\end{tabular}

weights, the air buoyancy correction, the repeatability of balance, the isotopic composition, the impurities, the reference gauge blocks and the repeatability of the diameter measurements.

In case of very thin boron deposits which cannot be characterized by difference weighing the areal density is determined by neutron time of flight measurements [15].

For tristearin deposits the hydrogen content is determined by combustion analysis according to the principle of catalytic tube combustion in an oxygenated atmosphere [23].

An overview of the techniques for the preparation and characterization of thin targets for neutron-induced reaction experiments is shown in Table 2.

\section{Examples of neutron-induced reactions}

\subsection{Neutron fluence monitors}

In neutron reaction cross-section measurements, the neutron fluence needs to be accurately determined. This neutron fluence is measured through the detection of charged particles that are produced in standard neutroninduced reactions. Depending on the neutron energy region of interest, a specific standard reaction is used. In the thermal and resonance energy region, ${ }^{3} \mathrm{He},{ }^{6} \mathrm{Li}$ and ${ }^{10} \mathrm{~B}$ are used to determine the neutron fluence in the beam. In the case of ${ }^{6} \mathrm{Li}$ and ${ }^{10} \mathrm{~B}$, the method is based on the detection of alpha particles produced in the $(n, \alpha)$ reactions ${ }^{6} \mathrm{Li}+\mathrm{n} \rightarrow{ }^{3} \mathrm{H}+\alpha$ and ${ }^{10} \mathrm{~B}+\mathrm{n} \rightarrow{ }^{7} \mathrm{Li}+\alpha$.

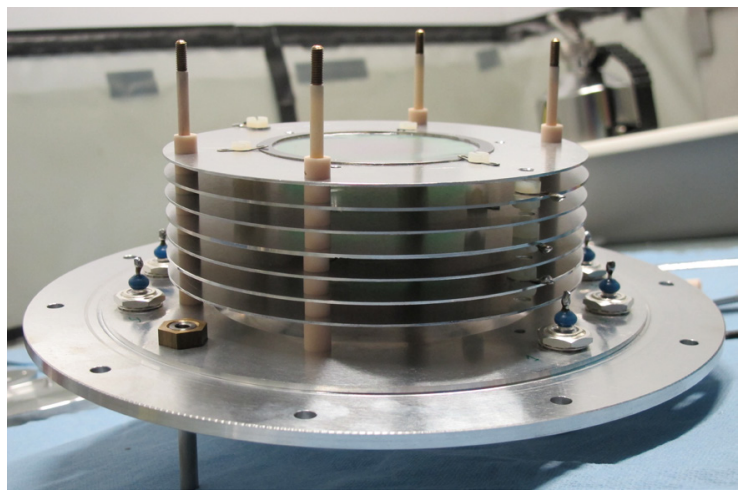

Fig. 4. Multi-layer ${ }^{235} \mathrm{U}$ based fluence monitor.
In the fast neutron energy region, fluence measurements are based on the detection of fission fragments produced in the standard ${ }^{235} \mathrm{U}(\mathrm{n}, \mathrm{f})$ reaction.

Typical detectors used for fluence measurements are gas-filled (e.g. ionisation chamber) and solid-state detectors or scintillators (e.g. Li-glass detector). In both cases, thin film layers are needed to limit energy loss of the reaction products within the layer. Fig. 4 shows the interior of a multi-layer ${ }^{235} \mathrm{U}$ based fluence monitor.

In the high-energy range, for mono-energetic neutrons up to $24 \mathrm{MeV}$, a recoil proton telescope can be used to measure the neutron fluence. It makes use of the $(\mathrm{n}, \mathrm{p})$ cross-section. The instrument consists of a tristearin $\left(\mathrm{C}_{57} \mathrm{H}_{110} \mathrm{O}_{6}\right)$ target as radiator to generate the protons and a silicon surface barrier detector as solid angle proton counter. The number of detected recoil protons is directly linked to the areal density of hydrogen in the tristearin target. The hydrogen content of the tristearin bulk and deposited material has been determined by combustion analysis. The stability of tristearin has been demonstrated even for long evaporation runs and a reliable value of the hydrogen content of 12.35 (10) wt\% has been established [23].

\subsection{Neutron-induced cross-section experiments}

For ${ }^{233} \mathrm{U}\left(\mathrm{n}, \mathrm{n}^{\prime} \gamma\right)$ cross-section measurements at GELINA [24] a $8.3 \mathrm{~g}{ }^{233} \mathrm{U}$ disc with a diameter of $30 \mathrm{~mm}$, a thickness of $0.64 \mathrm{~mm}$ and an activity of $3 \mathrm{GBq}$ has been prepared by punching (left part of Fig. 5). The (n,xn $\gamma$ ) cross-sections of actinides involved in new generation cycles like the thorium cycle, are important to test and improve predictive power of theoretical codes allowing a better knowledge of the neutron inelastic scattering process. The ${ }^{233} \mathrm{U}$ disc was prepared by punching a $76.2 \times 38.1 \times 0.64 \mathrm{~mm} \mathrm{U}$ foil in a glove box in the nuclear controlled area. Because of the limited ${ }^{233} \mathrm{U}$ material simulations have been done with $\mathrm{Al}$, stainless steel, $\mathrm{Ta}$ and $\mathrm{U}$ to find the optimum punching parameters. Finally the ${ }^{233} \mathrm{U}$ material bagged in a selfsealing bag and a sealed single PVC bag, was punched with the P/O Weber $300 \mathrm{kN}$ press at a force of 20-25 kN in combination with a die of $30 \mathrm{~mm}$ diameter. After weighing the ${ }^{233} \mathrm{U}$ disc, the target was transferred to an argon box for mounting between two Al foils with a thickness of $50 \mu \mathrm{m}$ each in a tailor-made holder that 
maintains the Ar atmosphere and allows placing it in a neutron beam of at most $55 \mathrm{~mm}$ diameter with minimal support material in the beam. The diameter of the $U$ disc has not been measured; instead the diameter of the punch die has been determined by measuring the diameter of two punched test samples.

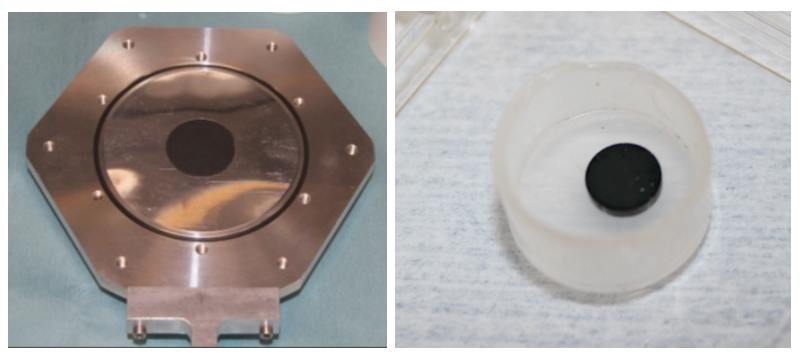

Fig. 5. Left part: ${ }^{233} \mathrm{U}$ disc with a diameter of $30 \mathrm{~mm}$ and thickness of $0.64 \mathrm{~mm}$ prepared by punching and mounted in a measurement holder with $50 \mu \mathrm{m}$ Al foil. Right part: Picture of a $5 \mathrm{~mm}$ diameter pellet of $0.1 \mathrm{~g}^{238} \mathrm{U}_{3} \mathrm{O}_{8}$ in a plastic container.

A ${ }^{238} \mathrm{U}_{3} \mathrm{O}_{8}$ pellet (right part of Fig. 5) with a mass of $0.1 \mathrm{~g}$, a diameter of $5 \mathrm{~mm}$ and with a low ${ }^{236} \mathrm{U} /{ }^{238} \mathrm{U}$ ratio of $7 \cdot 10^{-12}$ has been prepared with a Specac 15 Ton hydraulic press operating at $10 \mathrm{kN}$ and a $5 \mathrm{~mm}$ diameter die. The mass has been determined by weighing. The pellet is used in a campaign to measure the ${ }^{235} \mathrm{U}(\mathrm{n}, \gamma)$ cross-section. The cross-section will be deduced by quantifying the number of ${ }^{236} \mathrm{U}$ nuclei produced after neutron irradiation at FRM II (ForschungsNeutronenquelle Heinz Maier-Leibnitz) at the Technical University of Munich.

To investigate the mass, kinetic energy and nubar variations with the resonance energy and spin in the resolved resonance region of the ${ }^{239} \mathrm{Pu}$ fission crosssection, a ${ }^{239} \mathrm{Pu}$ layer with a diameter of $50 \mathrm{~mm}$ and an areal density of $29.95 \mu \mathrm{g} \cdot \mathrm{cm}^{-2}$ was prepared by vacuum evaporation on a polyimide foil of $38 \mu \mathrm{g} \cdot \mathrm{cm}^{-2}$. The importance of an excellent target to achieve an optimum mass and energy resolution over all emission angles is demonstrated in Fig. 6. The figure presents the fission fragment pulse height versus the cosine of the emission angle depicted for fission fragments detected in ${ }^{239} \mathrm{Pu}(\mathrm{n}, \mathrm{f})$ reactions obtained in a double-energy measurement with a twin Frisch-grid ionization chamber. The left part shows fragments emitted to the layer side, and the right part fragments emitted through the substrate in opposite direction. The distribution on the left side of Fig. 6 reflects the nearly perfect homogeneity of the active layer by a complete separation of light and heavy fragments irrespective of the emission angle. Also, the distribution obtained after passing the polyimide foil (right part of Fig. 6) shows still very good separation down to $\cos \theta=0.3$, which corresponds to an emission angle larger than $70^{\circ}$ relative to the beam axis. This high-quality pulse-height distribution allows obtaining fission fragment yield distributions with a mass resolution better than 5 mass units at FWHM. It is thanks to an excellent thin-layer target that fission crosssection and fragment yield measurements may be performed with a geometrical efficiency close to $2 \pi$.
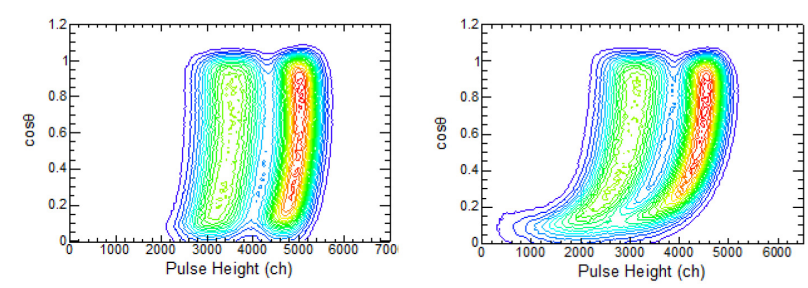

Fig. 6. Fission fragment pulse height versus the cosine of the fission axis relative to the axis of the incident neutron beam as obtained from the reaction ${ }^{239} \mathrm{Pu}(\mathrm{n}, \mathrm{f})$. Left part: pulse height after having passed the target layer, right part: pulse height after passing the target layer and the polyimide substrate.

\section{Investigations on-going}

\subsection{Molecular plating on Al backings with a thickness smaller than $1 \mu \mathrm{m}$}

The possibility to deposit thin layers of material on extremely thin backings by molecular plating is being investigated. The thin backings used for this purpose are made of Al foils of 6,2 and $0.8 \mu \mathrm{m}$ thickness mounted on $\mathrm{Al}$ rings of $0.5 \mathrm{~mm}$ thickness, $30 \mathrm{~mm}$ inner diameter and $40 \mathrm{~mm}$ outer diameter. The molecular plating cell has been re-designed to reduce the mechanical stress on the $\mathrm{Al}$ foil and minimize the occurrence of foil-break during the mounting and disassembling of the cell and during the molecular plating process.

\subsection{Production of recyclable powder pellets}

Investigation is on-going for the production of recyclable uranium and plutonium powder pellets prepared by cold compacting. In case of limited and valuable material, it is favourable to be able to recuperate the powder afterwards so that it can be used for other experiments. In this case mixing the powder with a binder like sulphur powder is not recommended as purification of the original powder can become complicated and time consuming especially in case of highly radioactive material. Tests are ongoing to find the optimum press parameters for different diameters and amount of material to produce mechanically stable $\mathrm{U}_{3} \mathrm{O}_{8}$ pellets and to set the boundaries of the preparation process.

\subsection{Cleaning of glass plates for polyimide foils}

Cleaning of glass plates used in the production process of polyimide foils is still time consuming and it is difficult to get the plates hydrophilic, i.e. the spreading of water on the surface of the glass plate should be uniform. Investigation is on-going to use oxygen plasma to "soft" etch the surface instead of cleaning the glass plates by rubbing with a paper towel and water as reported in ref. [13]. Several glass plates are cleaned in the plasma etching system under different cleaning conditions. They are covered with the polyimide solution and placed in the oven at $350^{\circ} \mathrm{C}$ for polymerization. The ability to release the foil from the glass plate is related to the surface of the glass plate. Tests are being conducted 
to find the optimum parameters for the plasma pod.

\subsection{Preparation of uranium deposits by e-beam evaporation}

In the frame of the replacement of the old ${ }^{235} \mathrm{U}$ evaporator, an investigation is on-going to prepare thin uranium layers by electron beam physical vapour deposition instead of evaporative deposition in which the material to be deposited is heated to a high vapour pressure by electrically resistive heating. The advantage would be that the original $\mathrm{U}_{3} \mathrm{O}_{8}$ material can be used instead of first converting this material into $\mathrm{UF}_{4}$ by a time consuming wet chemical precipitation method.

\subsection{Production of an evaporator}

Commercially available vacuum evaporators are not suitable to explore vacuum deposition parameters. A design for an evaporator has been made and the equipment, dedicated to stable isotopes, will be produced and used to better understand the vacuum deposition process.

\section{Conclusion}

For neutron-induced reaction measurements targets are produced according to the reaction being studied. Thick targets like discs and foils are prepared by cutting, rolling and punching and by cold compacting powder pellets. The targets are characterised for their mass by accurate weighing and for the area by accurate vision measuring microscopy. Thin deposits on a substrate are prepared by physical vapour deposition and molecular plating. The area of the deposits is determined by measuring the inner diameter of the mask during the deposition process. The mass of the stable deposits is determined by accurate weighing and of the actinide deposits via the activity measured by low-geometry alpha-particle counting.

The authors thank the JRC-Geel colleagues: the workshop for the production of the molecular plating cells, containers, masks etc., the nuclear chemistry and nuclear mass spectrometry group for the isotopic composition of the actinide material, Jan Heyse for the characterization of boron targets by $n_{-}$TOF measurements and the engineering materials laboratory for the SEM-EDX spectroscopy of stable targets; and the JRCKarlsruhe colleagues: the analytical service for the ICP-MS analysis, the surface science laboratory for the XPS and the electron microscopy group for the morphology and elemental composition analysis by SEM-EDX.

\section{References}

1. https://ec.europa.eu/jrc/en/research-topic/neutrondata

2. W. Mondelaers, et al., Il Nuovo Cimento 38 C, 175 (2015)

3. https://www.oecd-nea.org/science/nucleardata.html

4. http://www.oecd-nea.org/dbdata/hprl/

5. A. Stolarz, et al., Nucl. Instrum. Methods A 613, 351-356 (2010)

6. G. Sibbens, et al., in Proceedings of the Scientific Workshop on Neutron Measurements, Theory and Applications, Nuclear data for Sustainable Nuclear Energy, 28 - 30 April, 2009, Geel Belgium

7. R. Nolte, in Lecture notes at the Ecole Joliot Curie 2014 "Neutrons and Nuclei, 28.09-3.10.2014, Frejus, France

8. P. Schillebeeckx, et al., Nucl. Instrum. Methods A 613, 378-385 (2010)

9. A. Bail, et al., in AIP Conference Proceedings 1175, 2009, 4th International Workshop on Nuclear Fission and Fission-Product Spectroscopy, Cadarache, France

10. A. Göök, et al., Nucl. Instrum. Methods A 830, 366374 (2016)

11. S. Oberstedt, et al., Phys. Rev. C 90, 024618 (2014)

12. M.O. Frégeau, et al., Nucl. Instrum. Methods A 817, 35-41 (2016)

13. G. Sibbens et al., Nucl. Instrum. Methods A 655, 47-52 (2011)

14. G. Sibbens et al., J. Radioanal. Nucl. Chem. 299, 1093-1098 (2014)

15. D. Vanleeuw, et al., Preparation and characterization of ${ }^{10} \mathrm{~B}$ targets at JRC-Geel, these proceedings.

16. G. Sibbens et al., J. Radioanal. Nucl. Chem. 305, 723-726 (2015)

17. D. Vanleeuw, et al. in AIP Conference Proceedings 1962, 030008 (2018)

18. D. Vanleeuw et al., J. Radioanal. Nucl. Chem. 299, 1113-1120 (2014)

19. G. Sibbens et al., Appl. Rad. Isot. 87, 229-232 (2014)

20. G. Sibbens, et al., EPJ Web of Conferences 146, 04062, (2017)

21. G. Sibbens, et al., in AIP Conference Proceedings 1962, 030007 (2018)

22. J. Heyse et al., J. Radioanal. Nucl. Chem. 299, 1055-1059 (2014)

23. D. Vanleeuw et al., J. Radioanal. Nucl. Chem. 305, 957-962 (2015)

24. M. Kerveno et al., EPJ Web of Conferences 146, 11012 (2017) 